\title{
Statistical Geometry and Space-Time
}

\author{
Hans Grauert
}

Mathematisches Institut der Universität, D-3400 Göttingen, Federal Republic of Germany

\begin{abstract}
In this paper I try to construct a mathematical tool by which the full structure of Lorentz geometry to space time can be given, but beyond that the background - to speak pictorially - the subsoil for electromagnetic and matter waves, too. The tool could be useful to describe the connections between various particles, electromagnetism and gravity and to compute observables which were not theoretically related, up to now. Moreover, the tool is simpler than the Riemann tensor: it consists just of a set $S$ of line segments in space time, briefly speaking.
\end{abstract}

\section{The System $S$}

We first consider a global euklidian structure in the fourdimensional real number space $\mathbb{R}^{4}$. Later on, also local structures and curvature will be taken into account.

Let us denote the points of $\mathbb{R}^{4}$ by $\mathfrak{x}=\left(x_{0}, \ldots, x_{3}\right), \mathfrak{y}=\left(y_{0}, \ldots, y_{3}\right)$ and so on. The first component of the quadruples always is called the time coordinate. We put $\mathfrak{x}^{\prime}=\left(x_{1}, \ldots, x_{3}\right), \mathfrak{y}^{\prime}=\left(y_{1}, \ldots, y_{3}\right)$ and so forth. By $\delta(\mathfrak{x}, \mathfrak{y})$ a Radon measure in $\mathbb{R}^{4} \times \mathbb{R}^{4}$ is denoted which has the union of (full) light cones for support: $L=$ $\left\{(\mathfrak{x}, \mathfrak{y})=(\mathfrak{y}-\mathfrak{x})^{2}=0\right\}$, where $\mathfrak{z}^{2}=-z_{0}^{2}+z_{1}^{2}+z_{2}^{2}+z_{3}^{2}$ is the Lorentz norm. If $\mathfrak{f}$ is a test function in $\mathbb{R}^{4} \times \mathbb{R}^{4}$ then the following equation shall be valid:

$$
\int_{\mathbb{R} 4} \delta(\mathfrak{R}, \mathfrak{y}) \mathfrak{f}(\mathfrak{x}, \mathfrak{y})=\int_{L} d \mathfrak{x} d \mathfrak{y}^{\prime}\left\|\mathfrak{y}^{\prime}-\mathfrak{x}^{\prime}\right\|^{-1} \mathfrak{f}(\mathfrak{x}, \mathfrak{y})
$$

Here $\left\|\mathfrak{y}^{\prime}-\mathfrak{x}^{\prime}\right\|$ is the euclidean distance of $\mathfrak{y}^{\prime}$ and $\mathfrak{x}^{\prime}$ in $\mathbb{R}^{3}$. It is well known that the measure is invariant under all translations and all Lorentz-transformations (that means under all transformations of the Poincare group). Furthermore it is symmetric in $\mathfrak{x}$ and $\mathfrak{y}$ and on the other hand a linear transformation. which leaves it invariant belongs to the Poincaré group.

To prevent misunderstanding: I am far from asserting to have really solved any physical problem. This short paper gives some mathematical ideas, only, which might - I hope - prove to be helpful in future 
Now an intuitive geometrical (not mathematical) interpretation of $\delta(x, \mathfrak{y})$ is given. Let us think a set $S$ of (closed) oriented line segments in $\mathbb{R}^{4}$. Orientation means here that for each line segment of $S$ it is defined what the origin point and what the end point is. We think us $S$ of such kind that it has the following property: If $V_{1}, V_{2} \subset \mathbb{R}^{4}$ are bounded open subsets then the integral

$$
M\left(V_{1}, V_{2}\right)=\int_{V_{1} \times V_{2}} \delta(\mathfrak{x}, \mathfrak{y})
$$

is a measure for the "number" of line segments of $S$ running from $V_{1}$ to $V_{2}$. Of course, this number has to be infinite, in general. That is the reason why this interpretation of $\delta$ is not mathematically exact. Also the use of Lebesgues integral is not possible to come from the set of line segments of $S$ beginning in $V_{1}$ and ending in $V_{2}$ to the real number $M\left(V_{1}, V_{2}\right)$. There is no need for an exact mathematical interpretation. However, one way to do it, is the following:

Replace $S$ by a sequence of sets $S_{v}$ of line segments together with a sequence of real positive numbers $r_{v}$ converging to 0 such that:

1) $S_{v} \subset S_{v+1}$ always,

2) the number $n_{v}\left(V_{1}, V_{2}\right)$ of line segments of $S_{v}$ which pass from $V_{1}$ to $V_{2}$ always is finite,

3) $M\left(V_{1}, V_{2}\right)=\lim _{v \rightarrow \infty} r_{v} n_{v}\left(V_{1}, V_{2}\right)$.

And now the intuitive interpretation for $S=\cup S_{v}$ has obtained some mathematical meaning. Of course, everything would be nicer if $\mathbb{R}^{4}$ were a discrete space: if the number of line segments of $S$ from $V_{1}$ to $V_{2}$ always were big but finite and the set of points in $V_{1}$ and $V_{2}$, also, such that the whole object consists just of the segments and some points on them. Therefore, it may be that it is better to consider $\mathbb{R}^{4}$ as an idealisation of such a space, more precisely as a limit of line complexes (in the sense of topology) with distribution of lines becoming denser and denser.

Let us return to our intuitive $S$. We have $M\left(V_{1}, V_{2}\right)=M\left(V_{2}, V_{1}\right)$ : the density of lines of $S$ of given length is the same in both directions. From the definition of $\delta(\mathfrak{x}, \mathfrak{y})$ follows moreover that almost all line segments of $S$ are on a light cone and that each line (light ray) on a light cone can be approximated by line segments of $S$ : For each such line, each $R>0$, each point $P$ on that line and each neighborhood of that line there exists a line segment $s \in S$ in that neighborhood such that the origin of $s$ has in one direction and the end of $s$ in the other direction a distance of $P$ bigger than $R$.

We consider two sets of line segments to be equivalent if their distribution is given by the same Radon measure. We call an equivalence class a distribution of line segments. From now on $S$ denotes always such a distribution corresponding to the integral at the begin. We say that a bijective transformation $f: \mathbb{R}^{4} \rightarrow \mathbb{R}^{4}$ leaves $S$ invariant if there exists a set of line segments $A \in S$ such that each line segment $s \in A$ is mapped onto a line segment such that a set of line segments $B \in S$ is obtained.

If $f$ is a topological transformation $\mathbb{R}^{4} \rightarrow \mathbb{R}^{4}$ which leaves $S$ invariant then from approximation by line segments it follows that light rays are mapped onto light rays. By Borchers and Hegerfeld $[1,2] f$ has to be linear and as is said before belongs to the Poincaré group. Therefore, $S$ gives the Lorentz geometry. 


\section{Statistical Equilibrium}

In this section we prove that the distribution of the line segments of $S$ satisfies a statistical equilibrium. Moreover, the distribution is determined by the equilibrium, probably.

A priori we assume, that $S$ is invariant against translations (homogeneity of the space) and space-like rotations (isotropy of the space). Beyond this $S$ shall have the following properties:

1) All the segments out of a set $A \in S$ are on (the boundary of) a light cone.

2) Proportional to the magnitude of the density the following process takes place:

a) pairs of segments of equal direction, for which the end point of the first segment is equal to the origin of the second, connect to a new segment;

b) pairs of segments of opposite direction, for which again the end point of the first segment is equal to the origin of the second, pass over into a tripel of segments in conformity to the following diagram:

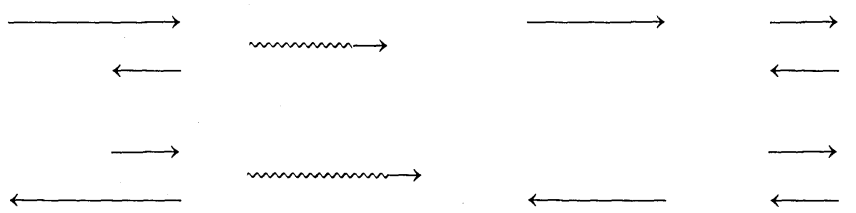

(of course, all segments are to be considered on a line).

The process b) means that the shorter segment causes the disconnection of the longer segment, if both segments have opposite direction.

Of course, we are building up just a mathematical theory. The line segments are purely mathematical objects and also the process is just a stochastic process in the mathematical sense. There is no physical interpretation at this point.

Because of the homogeneity and the isotropy of the space we can write:

$$
\delta(\mathfrak{x}, \mathfrak{y})=a\left(\left\|\mathfrak{y}^{\prime}-\mathfrak{x}^{\prime}\right\|\right) d \mathfrak{x} d \mathfrak{y}^{\prime} .
$$

Here $a(x)$ is a positive real function which is defined for $x>0$. It is clear that we have to integrate over $L$ instead of $\mathbb{R}^{4} \times \mathbb{R}^{4}$ if we write $\delta(\mathfrak{x}, \mathfrak{y})$ in this form. We obtain the right distribution of segments for the Lorentz geometry if $a(x)=1 / x$.

Let us consider a pair of points $(\mathfrak{x}, \mathfrak{y}) \in L$. The magnitude of building up of segments which lead from a volume element $d x$ around $x$ with the space component into a volume element $d \mathfrak{y}^{\prime}$ around $\mathfrak{y}^{\prime}$ by the process described under 2) is given by the following expression

$$
\operatorname{gen}(\mathfrak{x}, \mathfrak{y}) d \mathfrak{x} d \mathfrak{y}^{\prime}=\left(\int_{-\infty}^{+\infty} a(x-t) a(t) d t+4 a(x) \cdot \int_{x}^{\infty} a(t) d t\right) d \mathfrak{x} d \mathfrak{y}^{\prime} .
$$

Here $a(x)$ is continued by the equation $a(x)=a(-x)$ to the negative real numbers. In the expression for gen $(\mathfrak{x}, \mathfrak{y})$ both processes $2 \mathrm{a}$ ) and $2 \mathrm{~b}$ ) are taken into account. The integral from $-\infty$ to $+\infty$ contains the result of $2 a$ ) and the left segments in the result of $2 b)$. The second integral with the factor $4 a(x)$ contains all the segments 
at the right side of the results of process $2 \mathrm{~b}$ ). These segments are considered as to have same direction. This is admissible because the frequency of segments of same length with different direction is equal.

Let us consider now the magnitude of disintegration of segments which lead from the volume $d \mathfrak{x}$ around $\mathfrak{x}$ with the space component into the volume element $d \mathfrak{y}^{\prime}$ around $\mathfrak{y}^{\prime}$. We have to take in account the left sides of $2 \mathrm{a}$ ) and $2 \mathrm{~b}$ ). We obtain the following expression

$$
\operatorname{dis}(\mathfrak{x}, \mathfrak{y}) d \mathfrak{x} d \mathfrak{y}^{\prime}=\left(2 a(x) \int_{-\infty}^{+\infty} a(t) d t\right) d \mathfrak{x} d \mathfrak{y}^{\prime}
$$

Equilibrium means:

$$
\operatorname{gen}(\mathfrak{x}, \mathfrak{y})=\operatorname{dis}(\mathfrak{x}, \mathfrak{y}) \text {. }
$$

This is equivalent to the following equation

$$
\int_{-\infty}^{+\infty} a(t) a(x-t) d t=2 a(x) \int_{-x}^{+x} a(t) d t .
$$

The function $a(x)$ has to satisfy some conditions so that the equation is meaningful. We assume: $a(x)=a(-x), a(x)>0$ for $x \neq 0$. We furthermore assume that $a(x)$ is continuously differentiable for $x \neq 0$, that it is square integrable in infinity and that $h(x)=|x| \cdot a(x)$ is integrable in 0 .

The integrant on the right side of the equation in $t=0$ is

$$
2 a(x) \cdot a(t) \text {. }
$$

The integrant on the left side in $t=0$ is

$$
a(t) \cdot a(x)+\text { something integrable }
$$

and in $t=x$ is

$$
a(x) a(t-x)+\text { something integrable . }
$$

By integrating over an $\varepsilon$-interval around these points we get infinity [in the case $a(x)=1 /|x|$ for instance]. But on both sides of the equation of the same kind. So the equation stays meaningful even in the case, where $a(x)$ has a pole in 0 .

By a direct computation it can be proved that

$$
a(x)=\frac{c}{|x|}, \quad c>0
$$

are solutions of the integral equation. The expectation remains, that these are the only ones.

If this is the case then Lorentz geometry is determined by homogeneity, isotropy of $S$, the fact that all segments of $A \in S$ are on a light cone and the statistical equilibrium. If by a transformation light rays are mapped onto light rays then the transformation is linear, already, and belongs to a sum of the Poincare group and a group of dilatations. The system $S$ is needed to cancel the dilations. It gives a unit for measuring. 


\section{Vortices and Waves}

An essential tool of science in all directions are the waves of the different kinds. How is it possible for a space to oscillate locally? A Riemannian space can carry gravity waves. But how are the electromagnetic waves and how is the "infinity" of matter waves possible?

Actually, the motif for introducing $S$ is not to get another characterisation of Lorentz geometry. But the line segments of $(A \in) S$ form the subsoil of all these waves. The full theory was done in [3]. Here, only a brief outline shall be given. We restrict ourselves to the 2-dimensional case, that means to $\mathbb{R}^{2}$ with variables $x_{0}, x_{1}$. We assume that line segments can connect at the origin and at the end (at least). By such a connection the following "objects" are obtained, which are relevant to the theory:

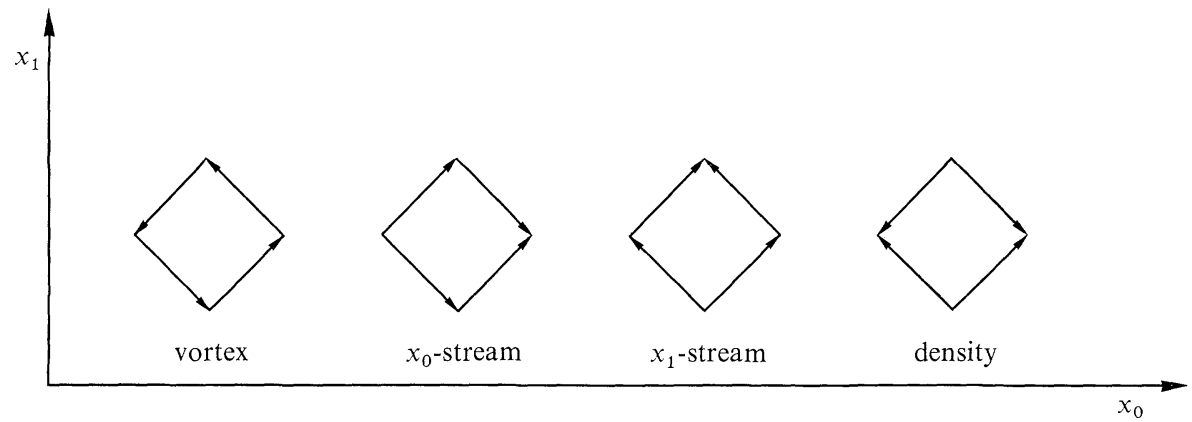

By Lorentz transformation more general objects can be generated out of these. All the shown objects are in positiv form. The negative ones are obtained by reversing the arrows. It is possible to form fields of objects (of the same kind with fixed length of line segments) by superposition of many of them. By this very smooth, only slowly changing fields can be obtained. The statistical equilibrium between small and big line segments effects also the objects. There is a equilibrium between small and big ones and also between the different kinds. This principal creates the waves. Consider the following skeleton diagram:

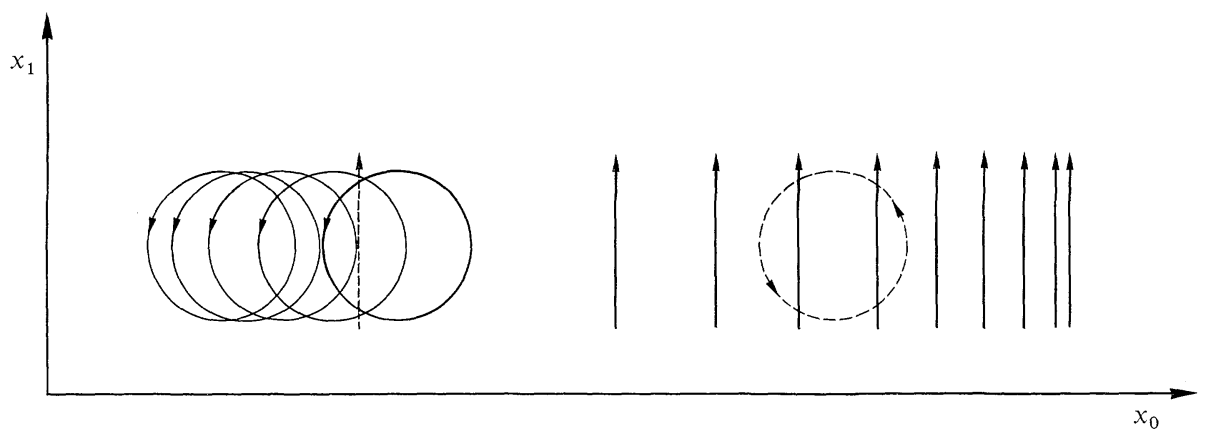

On the left side we have a field of positive vortices, on the right a field of $x_{1}$-streams. We assume that these fields - differently to the drawing - are in their magnitude independent of $x_{1}$. The magnitude $f=f\left(x_{0}\right)$ of the field of vortices is decreasing with time. A positive vortex has at its left side a negative stream, at its 
positive one a positive $x_{1}$-stream. The decreasing effects that not all the right streams will be cancelled by left streams of following vortices: so a positive $x_{1}$-stream of magnitude $g$ is generated in accordance with the equation:

$$
g=-f^{\prime} \text {. }
$$

On the right side of the diagram the magnitude $g$ of the $x_{1}$-stream is increasing with time. Now let us do an intuitive mathematical meditation. We see then: On the right side of the right part of the diagram there is a stronger "force" in $x_{1}$-direction than on the other. This seems to turn the subsoil around such that positive vortices are generated. In the exact theory this again is done by an equilibrium, see [3]. There also is shown that the magnitude $f$ of the generated field follows the law: $f=\omega^{2} g^{\prime}$. The whole two fields of vortices and streams are in equilibrium if $f$ (and similarly $g$ ) satisfies: $f^{\prime \prime}=-\omega^{2} f$ and the solutions are waves and it can be seen that the "mass" $\omega$ is proportional to the length of the line segments of the objects of the fields.

Thus a geometric interpretation of Dirac equation is obtained, moreover in [3] a geometric "unified field theory" which up to now, however, contains only the fields of electron, the neutrinos, photon and electric potential. By this the not observable magnitude of vacuum polarisation, the production of virtual photons is defined and a numerical expression for the elementary charge is obtained.

In the wave of a particle some of the line segments should be bound such that they enter the statistical equilibrium, no longer. This might be the reason for the fact that a particle causes a curvature of the space and so gravitation.

Because at all places several statistical equilibriums enter I like to call this new kind of geometry a statistical geometry.

Acknowledgement. I have to thank H. J. Borchers for valuable help.

\section{References}

1. Borchers, H.J., Hegerfeld,G.C.: Über ein Problem der Relativitätstheorie: Wann sind Punktabbildungen des $\mathbb{R}^{n}$ linear? Nachr. Akad. Wiss. Göttingen 10, 205-229 (1972)

2. Borchers, H.J., Hegerfeld, G.C.: The structure of space-time transformations. Commun. math. Phys. 28, 259-266 (1972)

3. Grauert,H.: Statistische Geometrie. Ein Versuch zur geometrischen Deutung physikalischer Felder. Nachrichten Akad. Wiss. Göttingen 1975 (to appear)

Communicated by R. Haag

Received October 13, 1975; in revised form January 22, 1976 